\title{
Mechanical versus bioprosthetic mitral valve replacement in patients $<65$ years old
}

Tsuyoshi Kaneko, MD, Sary Aranki, MD, Quratulain Javed, MD, Siobhan McGurk, BS, Prem Shekar, MD, Michael Davidson, MD, and Lawrence Cohn, MD

Objective: Because of its durability, the mechanical valve is typically chosen for young patients undergoing mitral valve replacement (MVR). However, a bioprosthetic valve might have the benefit of valve-in-valve transcatheter valve replacement when valve failure occurs. We examined the outcomes in patients who had undergone mechanical valve MVR (MVRm) versus bioprosthetic valve MVR (MVRb) in patients aged $<65$ years.

Methods: A total of 768 consecutive patients aged $<65$ years, who had undergone MVR from January 1991 to June 2012 were identified. Propensity matching was used to derive a case-control subset for analysis. Long-term outcomes were collected by chart review, routine patient follow-up, and query of the Social Security Death Index. The postoperative and long-term outcomes of interest included combined stroke and embolic events, reoperations, and mortality.

Results: Of 768 consecutive patients, 627 were in the MVRm and 141 in the MVRb group. Propensity score matching yielded a cohort of $125 \mathrm{MVRb}(89 \%)$ and 125 control MVRm patients with similar etiology mixes. The groups were similar in age (MVRm, $53.2 \pm 9.0$ years; MVRb, $53.8 \pm 10.6$ years; $P=.617$ ) and other preoperative characteristics. The postoperative outcomes were also similar between the 2 groups, including reoperation for bleeding, stroke, deep sternal infection, sepsis, and length of hospital stay. The operative mortality was also similar (MVRm, 5.6\%; MVRb, $8.0 \% ; P=.617$ ). However, Kaplan-Meier analysis showed the MVRb group had a greater reoperation rate $(P=.001)$ and shorter estimated survival (11.3 vs 13.5 years, $P=.004)$. The incidence of bleeding and stroke or embolic events between the 2 groups was similar.

Conclusions: In the present report, MVRb for patients $<65$ years old was associated with a high reoperation rate and decreased survival. Although a future transcatheter valve-in-valve technique for a failed bioprosthetic valve might reduce the risk of reoperation, this finding confirms the safety of mechanical valves in this group. (J Thorac Cardiovasc Surg 2014;147:117-26)

The momentum toward the use of bioprosthetic valves for mitral valve replacement (MVR) has been great. From the Society of Thoracic Surgeons database, usage of the mechanical valve has decreased from $68 \%$ in 2000 to $37 \%$ in $2007 .{ }^{1}$ The median age of the patients receiving bioprosthetic valves has decreased significantly from 77 in 2000 to 71 in 2007, and this trend is likely to continue. Classic teaching has recommended mechanical valves for younger patients to avoid structural valve deterioration (SVD) at the expense of lifetime anticoagulation. However, the lower SVD rate with current bioprostheses, the lower risk of reoperation, and young patients' decision to not

From the Department of Cardiac Surgery, Brigham and Women's Hospital, Boston, Mass.

Disclosures: Authors have nothing to disclose with regard to commercial support.

Read at the 39th Annual Meeting of The Western Thoracic Surgical Association, Coeur d'Alene, Idaho, June 26-29, 2013.

Received for publication June 26, 2013; revisions received July 29, 2013; accepted for publication Aug 14, 2013; available ahead of print Sept 30, 2013.

Address for reprints: Lawrence Cohn, MD, Department of Cardiac Surgery, Brigham and Women's Hospital, 75 Francis St, Boston, MA 02115 (E-mail: 1cohn@ partners.org).

$0022-5223 / \$ 36.00$

Copyright $(2014$ by The American Association for Thoracic Surgery

http://dx.doi.org/10.1016/j.jtcvs.2013.08.028 accept the activity constraints associated with anticoagulants have all contributed to the recent lower age cutoff for the use of bioprostheses. ${ }^{2}$

For MVR, the American College of Cardiology/American Heart Association guideline from 2008 has provided no age criteria for the class I recommendation. The class IIa recommendations are to use the mechanical valve in patients aged $<65$ years with atrial fibrillation. The bioprosthetic valve has been recommended for patients aged $<65$ years who are in sinus rhythm and who have elected to receive this valve for lifestyle considerations after detailed discussions of the risks of anticoagulation versus the likelihood that a second MVR might be necessary in the future. ${ }^{3}$ The European Society of Cardiology and the European Association for Cardio-Thoracic Surgery guideline has recommended the mechanical valve for those aged $<65$ years for MVR. ${ }^{4}$

Two randomized control studies were conducted in the 1970s to assess the outcomes between mechanical and bioprosthetic valves in the mitral position. Neither study showed any difference in survival. ${ }^{5,6}$ However, these studies did not focus on any specific age group. Few data exist on the specific group $<65$ years old when comparing the outcomes of mechanical versus bioprosthetic valves in 


\section{Abbreviations and Acronyms \\ EMRs = electronic medical records \\ MVR = mitral valve replacement \\ SVD $=$ structural valve deterioration \\ TMVR $=$ transcatheter mitral valve replacement \\ VIV $=$ valve-in-valve}

MVR. With the recent enthusiasm of valve-in-valve (VIV) transcatheter MVR (TMVR), the trend of patients receiving a bioprosthetic valve at a younger age will likely accelerate. Therefore, it is critical to understand the outcomes of receiving a mechanical versus bioprosthetic valve for MVR at a younger age.

The aim of the present study was to review the immediate and long-term outcomes of mechanical versus bioprosthetic MVR in patients $<65$ years old to provide information regarding valve selection in this age group.

\section{METHODS}

\section{Patient Selection}

A retrospective review of the hospital electronic medical records (EMRs) was conducted to identify all MVRs performed with a mechanical (MVRm) or stented bioprosthetic (MVRb) valve from January 1991 to June 2012 in patients aged $<65$ years old. Patients who had undergone multiple surgeries were included in the present study if they were $<65$ years old. A total of 768 patients underwent MVR within the study period. Patients who had undergone any type of previous cardiac surgery were included in the cohort. The exclusion criteria included any MVR performed in patients $>65$ years; no exclusions were made on gender, race, or other concomitant cardiac surgery. Our internal review board approved the present study.

\section{Data Collection}

The patient characteristics, medications, laboratory values, and inhospital outcomes of the index surgery were collected at presentation and extracted from the patients' EMRs. Mortality data, including the cause of death, were collected from the following sources: the Social Security Death Index, EMRs, and the Registry of Vital Records and Statistics, Department of Public Health (Dorchester, Mass). The long-term outcomes were extracted from a chart review of the EMRs, our internal outcomes data repository, and routine patient follow-up findings. We had $100 \%$ follow-up at 30 days and $99.1 \%$ long-term follow-up using our various sources.

The patient demographics and hospital outcomes were coded and defined according to the Society for Thoracic Surgeons Adult Cardiac Surgery database specifications, version 2.52 . Our primary short-term outcomes of interest were 30-day mortality, postoperative stroke, and re-exploration for bleeding. The secondary outcomes of in-hospital cardiac arrest, complete heart block, duration of ventilator use, intensive care unit length of stay, and hospital length of stay were also assessed. The long-term outcomes of interest were mortality, strokes and embolic events, major bleeding events, and valve-related reoperations. Major bleeding events were defined as presentations to a clinical office or emergency room for bleeding, bleeding that required medical intervention, and any unexpected or excessive bleeding as a complication of a procedure or other clinical intervention. The interval to a long-term event was calculated in months from the date of surgery to the first documented qualifying event, or if none occurred, to March 31, 2013.

\section{Statistical Analysis}

Normally distributed continuous variables are presented as the mean \pm standard deviation. Non-normally distributed continuous variables are presented as the median and interquartile range. Analyses of continuous variables were done using Student's $t$ test with Levine's homogeneity of variance or the Mann-Whitney $U$ test, as appropriate. Dichotomous variables were evaluated using Fisher's exact test and are presented as numbers and percentages. Survival and the interval to outcomes of interest were analyzed using the Kaplan-Meier method and life table estimations. Multivariate analysis of long-term survival was done using a forward stepwise Cox regression. For this analysis, we selected variables according to their clinical significance, variability across study cohorts, and known contributions to life expectancy. Interaction terms were examined. All statistical analyses were done using the Statistical Package for Social Sciences, version 13.0 (SPSS, Chicago, Ill) with $P \leq .05$ as the criterion for significance.

\section{Propensity Matching}

We conducted a matched group analysis using propensity matched cases of MVRb and MVRm. Propensity scores were generated using logistic regression analyses in 2 steps. Potential predictors were selected from a published data review, known confounding covariates for the outcomes of interest, differences between the 2 patient groups (Table 1), and clinical judgment. A forward-stepwise regression analysis was conducted, including examinations for interaction effects. An interaction variable between the surgeon and year of surgery was also examined to control for variability in case mix and surgical practice over time. Any variable with $P \leq .15$ was entered into an enter-method logistic regression analysis. The resulting adjusted predicted probability for each patient was then used to select matched groups according to probability scores of $<.01$ (a priori algorithm). This final model was moderately robust (area under the curve, 0.734; 95\% confidence interval, 0.686-0.782; $P<.001$ ) and contained age (in years), history of hypercholesterolemia, hypertension, renal failure, active endocarditis, ischemic disease, operative status (elective, urgent, or emergent) perfusion time (in minutes), and year of surgery. The resultant score was used to match the MVRm controls to MVRb cases with an equal or nearest probability score (within 0.01 ). Overall, $125 \mathrm{MVRb}$ cases were matched $1: 1$ to $125 \mathrm{MVRm}$ controls $(88.7 \%)$.

\section{RESULTS \\ Total Cohort}

Of 768 consecutive patients, 627 had undergone MVRm and $141 \mathrm{MVRb}$. The median follow-up period was 7 years (8 years for MVRm and 3 years for MVRb), for a total of 5899 patient-years during the study period. The characteristics and in-hospital outcomes are listed in Table 1.

The patients in the MVRb group were older $(54.3 \pm 10.3$ years vs $51.8 \pm 9.7$ years, $P=.005$ ) and were more likely to have a history of renal failure (25 of 414 vs 62 of 627 , $P=.012)$. The remaining characteristics were substantially similar regarding the proportion of women, left ventricular ejection fraction, New York Heart Association class, and cardiac surgery history.

Both groups had similar cardiac history. Of the MVRm and MVRb groups, $6.4 \%$ (40 of 627) and 8.5\% (12 of 141) had undergone previous coronary artery bypass grafting (CABG, $P=.356), 18.8 \%$ (118 of 627) and $18.4 \%$ (26 of 141) had undergone previous valve surgery $(P=1.000)$, and $1.8 \%$ (11 of 627$)$ and $5.0 \%$ (7 of 141$)$ 
TABLE 1. Characteristics and in-hospital outcomes for whole cohort

\begin{tabular}{|c|c|c|c|}
\hline Variable & $\begin{array}{c}\text { MVRm } \\
(\mathrm{n}=627)\end{array}$ & $\begin{array}{c}\text { MVRb } \\
(n=141)\end{array}$ & $\begin{array}{c}P \\
\text { value } \\
\end{array}$ \\
\hline \multicolumn{4}{|l|}{ Preoperative data } \\
\hline Age (y) & $51.8 \pm 9.7$ & $54.3 \pm 10.3$ & $\leq .005$ \\
\hline Female gender & $57.3(359)$ & $55.3(78)$ & $\leq .707$ \\
\hline Renal failure & $9.9(62)$ & $17.7(25)$ & $\leq .012$ \\
\hline $\begin{array}{l}\text { Preoperative creatinine } \\
\qquad(\mathrm{mg} / \mathrm{dL})\end{array}$ & $1.31 \pm 1.5$ & $1.34 \pm 1.1$ & $\leq .799$ \\
\hline Ejection fraction $(\%)$ & $60(50-65)$ & $60(50-65)$ & $\leq .767$ \\
\hline NYHA class III-IV & $54.1(339)$ & $52.5(74)$ & $\leq .779$ \\
\hline Previous CABG & $6.4(40)$ & $8.5(12)$ & $\leq .356$ \\
\hline Previous valve surgery & $18.8(118)$ & $18.4(26)$ & $\leq 1.000$ \\
\hline $\begin{array}{l}\text { Previous CABG and valve } \\
\text { surgery }\end{array}$ & $1.8(11)$ & $5.0(7)$ & $\leq .320$ \\
\hline \multicolumn{4}{|l|}{ Etiology } \\
\hline Active endocarditis & $8.5(53)$ & $14.2(20)$ & $\leq .055$ \\
\hline Healed endocarditis & $3.0(19)$ & $2.1(3)$ & $\leq .781$ \\
\hline Rheumatic & $40.2(252)$ & $31.2(44)$ & $\leq .055$ \\
\hline Myxomatous & $15.8(99)$ & $12.8(18)$ & $\leq .437$ \\
\hline Ischemic & $7.3(46)$ & $14.2(20)$ & $\leq .012$ \\
\hline $\begin{array}{l}\text { Nonischemic } \\
\text { cardiomyopathy }\end{array}$ & $1.9(12)$ & $0.7(1)$ & $\leq .481$ \\
\hline Other & $23.3(146)$ & $24.8(35)$ & $\leq .742$ \\
\hline \multicolumn{4}{|l|}{ Operative data } \\
\hline Minimally invasive & $5.9(37)$ & $2.8(4)$ & $\leq .211$ \\
\hline Concomitant CABG & $18.3(115)$ & $22.0(31)$ & $\leq .342$ \\
\hline Perfusion time (min) & $158(116-217)$ & $169(121-217)$ & $\leq .158$ \\
\hline Crossclamp time (min) & $109(75-158)$ & $117(87-163)$ & $\leq .050$ \\
\hline \multicolumn{4}{|l|}{ Postoperative outcomes } \\
\hline Reoperation for bleeding & $4.6(29)$ & $5.0(7)$ & $\leq .827$ \\
\hline Redo valve & $0.5(3)$ & $0.0(0)$ & $\leq 1.000$ \\
\hline Permanent stroke & $2.2(14)$ & $5.7(8)$ & $\leq .044$ \\
\hline DSWI & $0.6(4)$ & $2.1(3)$ & $\leq .120$ \\
\hline Sepsis & $1.1(7)$ & $3.5(5)$ & $\leq .051$ \\
\hline MSOF & $0.6(4)$ & $2.8(4)$ & $\leq .042$ \\
\hline $\operatorname{LOS}(\mathrm{d})$ & $8(6-13)$ & $9(6-17)$ & $\leq .205$ \\
\hline Operative mortality & $4.5(28)$ & $8.5(12)$ & $\leq .059$ \\
\hline Estimated survival (y) & 16.2 & 10.6 & $\leq .001$ \\
\hline
\end{tabular}

Data presented as mean \pm standard deviation, $\%$ (n), or median (interquartile range). $M V R m$, Mitral valve replacement with mechanical valve; $M V R b$, MVR with bioprosthetic valve; NYHA, New York Heart Association; $C A B G$, coronary artery bypass grafting; $D S W I$, deep sternal wound infection; $M S O F$, multisystem organ failure; $L O S$, length of stay.

had undergone previous CABG and valve surgery simultaneously, respectively.

The indications for surgery were different between the 2 groups. The MVRb patients were more likely to have active endocarditis (MVRb vs MVRm, 20 of 141 vs 53 of 627, respectively; $P=.055)$ or an ischemic etiology (MVRb vs MVRm, 20 of 141 vs 46 of 627, respectively; $P=.012)$ as an indication for surgery. In contrast, rheumatic disease was more frequent in the MVRm group than in the MVRb group (252 of 627 vs 44 of 141 , $P=.055)$.

The operative data showed a longer crossclamp time for MVRb (109 vs 117 minutes, $P=.05$ ) but a similar perfusion
TABLE 2. Implanted bioprosthetic valve type

\begin{tabular}{lc}
\hline \multicolumn{1}{c}{ Bioprosthetic valve type } & $\mathbf{n}(\%)$ \\
\hline Biocor & $28(19.9)$ \\
Carpentier Edwards Pericardial & $50(35.5)$ \\
Carpentier Edwards Porcine & $6(4.3)$ \\
Epic & $9(6.1)$ \\
Hancock & $24(17.0)$ \\
Hancock II & $3(2.1)$ \\
Magna Ease & $10(7.1)$ \\
Mosaic Porcine & $11(7.8)$ \\
Total & $141(100)$ \\
\hline Biocor and Epic, St Jude Medical, St Paul, Minn; Carpentier Edwards Pericardial and \\
Porcine, Magna Ease, Edwards Lifesciences, Irvine, Calif; Hancock, Hancock II, and \\
Mosaic Porcine, Medtronics, Minneapolis, Minn.
\end{tabular}

time (158 vs 169 minutes, $P=.158$ ). Of the MVRm and MVRb patients, $18.3 \%$ (115 of 627) and $22.0 \%$ (31 of 141) patients had undergone concomitant $C A B G$ at surgery $(P=.342)$. The type of bioprosthetic valves implanted is listed in Table 2. The most commonly used valve was the Carpentier Edwards Pericardial valve (Edwards Lifesciences, Irvine, Calif) in 50 patients $(35.5 \%)$ followed by the Biocor valve (St Jude Medical, St Paul, Minn) in 28 patients $(19.9 \%)$. Hancock I valves (Medtronics, Minneapolis, Minn) were used from 1992 to 2000 and were implanted in 24 patients $(17.0 \%)$.

Postoperatively, the MVRb group experienced significantly more strokes ( 8 of 141 vs 14 of $627, P=.044$ ) and a greater incidence of sepsis (5 of 141 vs 7 of 627 , $P=.044$ ) and multisystem organ failure (4 of 141 vs 4 of $627, P=.042)$. Operative mortality was also greater in the MVRb group $(8.5 \%, 12$ of $141 \mathrm{MVRb}$ vs $4.5 \%, 28$ of 627 MVRm, $P=.059)$. The estimated postoperative survival was shorter in the MVRb than in the MVRm group (median, 10.6 years vs 16.2 years, respectively; $P<.001$ ).

We were able to identify the long-term medication list for 132 of the 141 patients $(93.6 \%)$ in the MVRb group. We found that 34 of the 132 patients $(25.8 \%)$ were taking warfarin in the long term. Postoperative new-onset atrial fibrillation was seen in $15.8 \%$ of the MVRm and $18.4 \%$ of the MVRb groups.

\section{Propensity Matched Analysis}

Because of the inherent differences between the patient populations, we sought to create a case-control analysis using propensity matched cases. The characteristics and outcomes for the $125 \mathrm{MVRb}$ cases and $125 \mathrm{MVRm}$ controls are listed in Table 3. Statistically significant differences in the whole group, such as age, preoperative renal failure, endocarditis, and crossclamp time, were all matched. Overall, these groups were substantially similar in age, preoperative medical history, disease etiology, cardiac history, and operative outcomes.

After correction for these characteristics, the incidence of previously different in-hospital postoperative 
TABLE 3. Characteristics and in-hospital outcomes for 250 matched patients

\begin{tabular}{|c|c|c|c|}
\hline Variable & $\begin{array}{c}\text { MVRm } \\
(\mathbf{n}=125)\end{array}$ & $\begin{array}{c}\text { MVRb } \\
(\mathbf{n}=\mathbf{1 2 5})\end{array}$ & $\begin{array}{c}P \\
\text { value }\end{array}$ \\
\hline \multicolumn{4}{|l|}{ Preoperative data } \\
\hline Age $(y)$ & $53.2 \pm 9.0$ & $53.8 \pm 10.6$ & $\leq .617$ \\
\hline Female gender & $60.8(49)$ & $53.6(43)$ & $\leq .307$ \\
\hline Renal failure & $20.0(16)$ & $16.0(13)$ & $\leq .511$ \\
\hline $\begin{array}{l}\text { Preoperative creatinine } \\
\quad(\mathrm{mg} / \mathrm{dL})\end{array}$ & $1.36 \pm 1.2$ & $1.34 \pm 1.2$ & $\leq .888$ \\
\hline Ejection fraction $(\%)$ & $60(50-65)$ & $60(50-65)$ & $\leq .767$ \\
\hline NYHA class III-IV & $55.2(44)$ & $51.2(41)$ & $\leq .612$ \\
\hline Previous CABG & $3.2(4)$ & $4.9(5)$ & $\leq 1.000$ \\
\hline Previous valve surgery & $24.0(30)$ & $13.6(17)$ & $\leq .520$ \\
\hline $\begin{array}{l}\text { Previous CABG and valve } \\
\text { surgery }\end{array}$ & $2.4(3)$ & $4.0(5)$ & $\leq .722$ \\
\hline \multicolumn{4}{|l|}{ Etiology } \\
\hline Endocarditis & $12.8(10)$ & $12.8(10)$ & $\leq 1.000$ \\
\hline Ischemic & $12.0(10)$ & $12.8(10)$ & $\leq 1.000$ \\
\hline \multicolumn{4}{|l|}{ Operative data } \\
\hline Concomitant CABG & $18.4(15)$ & $23.2(19)$ & $\leq .342$ \\
\hline Perfusion time (min) & $162(113-220)$ & $170(121-215)$ & $\leq .691$ \\
\hline Crossclamp time (min) & $112(79-161)$ & $114(86-161)$ & $\leq .733$ \\
\hline \multicolumn{4}{|l|}{ Postoperative outcomes } \\
\hline Reoperation for bleeding & $4.8(4)$ & $5.6(4)$ & $\leq 1.000$ \\
\hline Redo valve & $0.0(0)$ & $0.0(0)$ & \\
\hline Permanent stroke & $2.4(2)$ & $6.4(5)$ & $\leq .216$ \\
\hline DSWI & $0.8(1)$ & $2.4(2)$ & $\leq .622$ \\
\hline Sepsis & $2.4(2)$ & $1.6(1)$ & $\leq 1.000$ \\
\hline MSOF & $0.8(1)$ & $2.4(2)$ & $\leq .622$ \\
\hline $\operatorname{LOS}(d)$ & $9(7-14)$ & $9(6-16)$ & $\leq .817$ \\
\hline Operative mortality & $5.6(4)$ & $8.0(6)$ & $\leq .617$ \\
\hline Estimated survival (y) & $13.5(0.7)$ & $11.3(1.0)$ & $\leq .004$ \\
\hline
\end{tabular}

Data presented as mean \pm standard deviation, $\%$ (n), or median (interquartile range). $M V R m$, Mitral valve replacement with mechanical valve; $M V R b$, MVR with bioprosthetic valve; $N Y H A$, New York Heart Association; $C A B G$, coronary artery bypass grafting; $D S W I$, deep sternal wound infection; $M S O F$, multisystem organ failure; $L O S$, length of stay.

complications, including stroke, sepsis, deep sternal infection, and multisystem organ failure, was similar between the 2 groups. Operative mortality was similar after correction $(5.6 \%$, MVRm and $8.0 \%, \mathrm{MVRb} ; P=.617)$. However, the long-term survival differences persisted, with an estimated median survival of 11.3 years for MVRb patients and 13.7 years for MVRm patients $(P=.004)$.

\section{Long-Term Outcomes}

Stroke or embolic event. Figure 1 shows the KaplanMeier analysis of the long-term risk of stroke or an embolic event. A total of 46 stroke or embolic events occurred. Of these, 40 were in the MVRm group and 6 in the MVRb group. The estimated freedom from stroke and embolic events at 5, 10, and 15 years was $95.3 \%, 93.2 \%$, and $90.7 \%$ for the MVRm group and $93.7 \%, 87.6 \%$, and $87.6 \%$ for the MVRb group, respectively. No differences were seen between the MVRm and MVRb patients after a follow-up period of 240 months $(P=.912)$.

Bleeding events. Figure 2 shows the Kaplan-Meier estimation of reported bleeding events. The estimated freedom from major bleeding at 5, 10, and 15 years was $87.2 \%$, $79.2 \%$, and $71.2 \%$ for the MVRm group and $91.1 \%$, $85.0 \%$, and $77.9 \%$ for the MVRb group, respectively. Although a greater cumulative proportion of MVRm patients experienced a bleeding event, the difference was not statistically significant $(P=.196)$.

Reoperations. Figure 3 shows the Kaplan-Meier curves for valve-related reoperations. The freedom from reoperation was estimated at $97.7 \%, 96.6 \%$, and $96.1 \%$ for the MVRm group and $96.6 \%, 86.6 \%$, and $75.3 \%$ for the MVRb group at 5, 10, and 15 years, respectively. The risk of reoperation was significantly greater for the MVRb patients $(P=.003)$.

Long-term survival. Figure 4 shows the long-term survival in the total and matched cohorts. The estimated $5-, 10-$, and 15 -year survival was $82.1 \%, 68.5 \%$, and $54.1 \%$ for the MVRm patients and $65.8 \%, 55.0 \%$, and $38.5 \%$ for the MVR patients, respectively $(P<.001)$. Even when the patient characteristics were controlled for in the propensity matched group, the survival differences persisted, with an estimated 5-, 10-, and 15-year survival of $83.4 \%, 69.2 \%$, and $62.6 \%$ in the MVRm group and $67.3 \%, 57.6 \%$, and $40.4 \%$ in the MVRb group $(P=.004)$.

Cox regression analysis. We conducted a Cox regression analysis to evaluate the predictors of long-term survival using the total study cohort (Table 4). Increasing age $(P \leq .001)$, perfusion time $(P \leq .001)$, and elevated creatinine $(P \leq .001)$ all increased the risk of mortality. Previous CABG or valve surgery $(P=.017$ and $P=.026$, respectively), urgent or emergent surgical status (vs elective, $P=.006$ ), and a history of congestive heart failure $(P=.002)$ or hypercholesterolemia $(P=.05)$ also increased the risk of mortality. When controlling for such factors, our multivariate analysis revealed that mortality was elevated for the MVRb cohort compared with the MVRm cohort (hazard ratio, 1.476; 95\% confidence interval, 1.073-2.031, $P=.017)$. Other variables tested, such as preoperative low ejection fraction, endocarditis, female gender, New York Heart Association class III and IV, a history of cerebrovascular accident, high body mass index, ischemic etiology, body surface area, and hypertension, were not associated with mortality $(P>.05)$.

\section{DISCUSSION}

In patients $>65$ years old, the use of bioprosthetic valves for MVR has been supported by the low rate of SVD and hemorrhagic complications. ${ }^{7,8}$ However, for patients $<65$ years old, bioprosthetic valves have experienced earlier calcification, leading to reoperation and limited durability. ${ }^{9}$ The decision of valve choice must be made by 


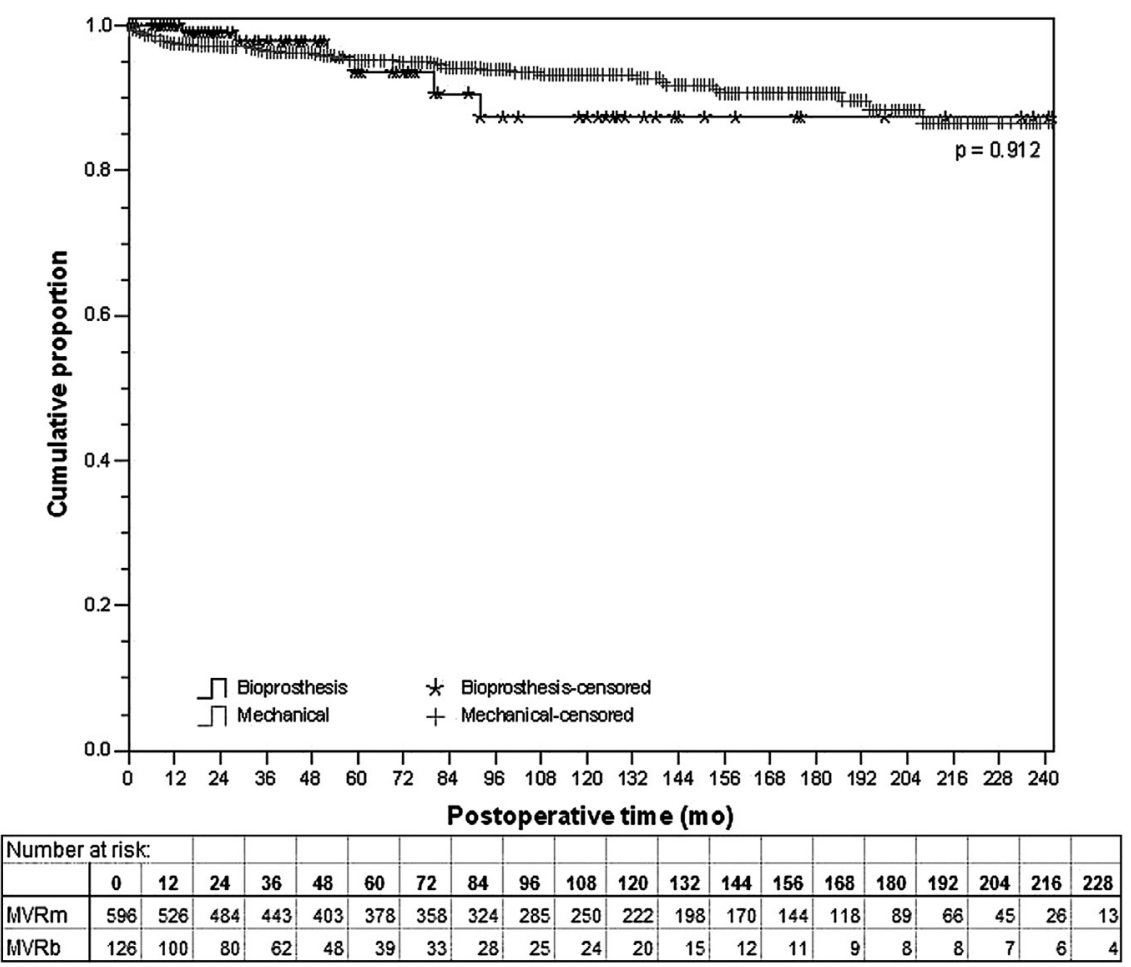

FIGURE 1. Kaplan-Meier curve of stroke and embolic events. $M V R m$, Mitral valve replacement with mechanical valve; $M V R b$, MVR with bioprosthetic valve.

balancing this risk with the risk of lifetime anticoagulation required with mechanical valves and the patient's lifestyle in younger patients.
The question "which valve has a better outcome in patients <65 years who are undergoing MVR" remains controversial. Only 2 randomized control studies regarding

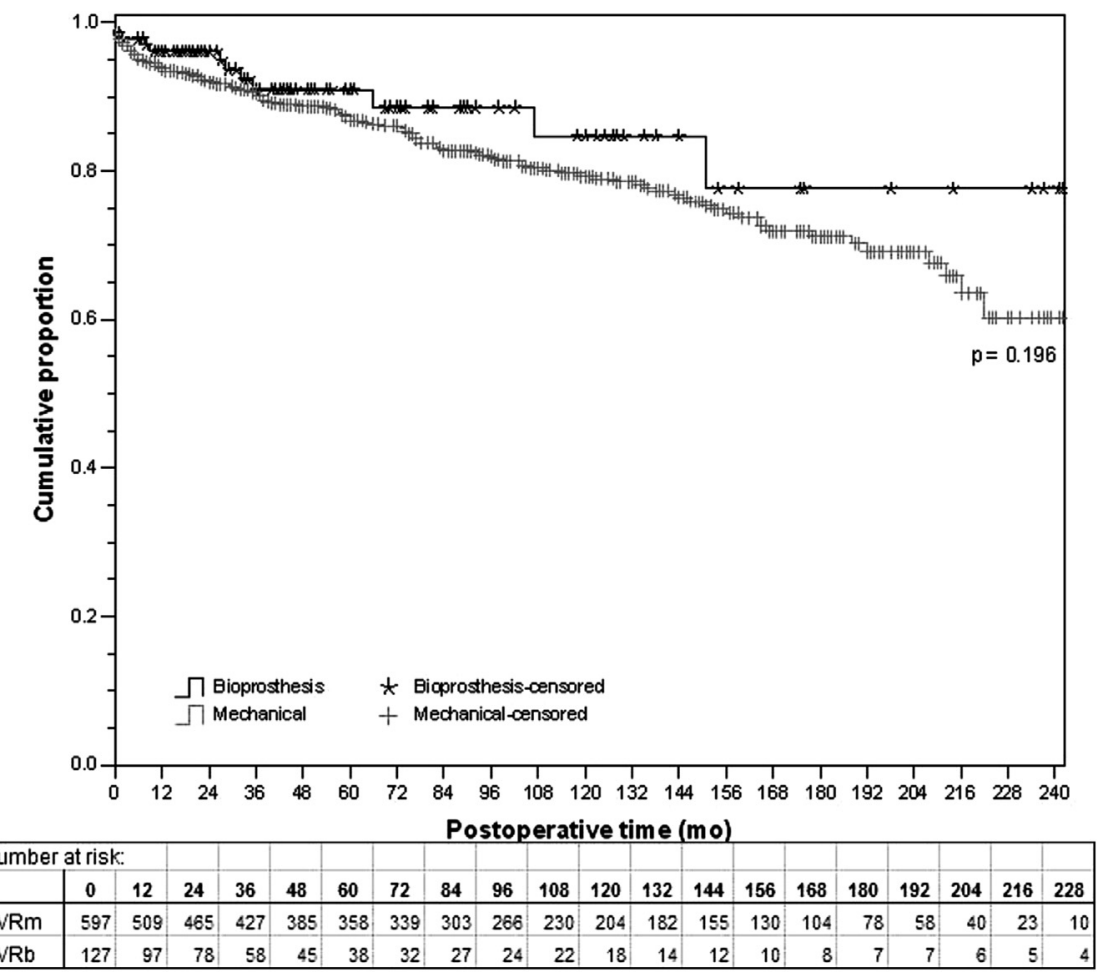

FIGURE 2. Kaplan-Meier curve of bleeding events. $M V R m$, Mitral valve replacement with mechanical valve; $M V R b$, MVR with bioprosthetic valve. 


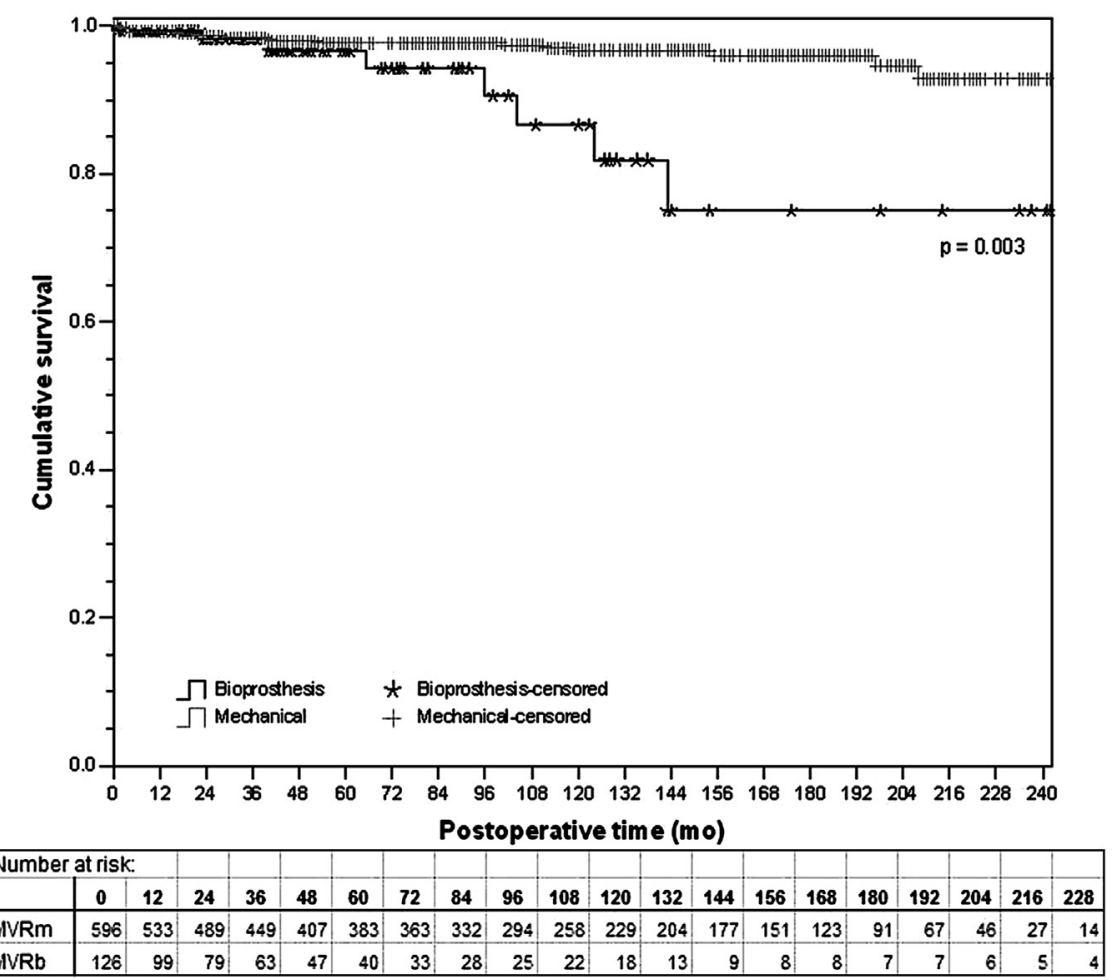

FIGURE 3. Kaplan-Meier curve of reoperation of mitral valve. $M V R m$, Mitral valve replacement with mechanical valve; $M V R b$, MVR with bioprosthetic valve.

mechanical valves versus bioprosthetic valves in the mitral position have been conducted. The Edinburgh study ${ }^{5}$ and the Veterans Affairs study ${ }^{6}$ from the 1970s showed similar survival after 20 and 15 years of follow-up, respectively. Both showed a greater incidence of bleeding complications with mechanical valves and a greater reoperation rate with bioprosthetic valves. Although the findings from these studies are important, they examined older generation prostheses, and no outcome was reported for a specific age group.

The present study was the 1 of the largest retrospective studies to date on this subject. The main finding of our study was that MVRb group $<65$ years old had an increased reoperation rate and shorter long-term survival, even after the propensity matched analysis. The incidence of bleeding and stroke and embolic events was similar between the 2 groups. These finding are different from the 2 previous randomized control studies and recent retrospective studies that reported similar survival between those receiving mechanical and biologic prostheses. ${ }^{10,11}$

\section{Survival}

The reported 10-year survival after MVR for those $<65$ years has been $65 \%$ to $78 \%$ with bioprosthetic and $60 \%$ to $76 \%$ with mechanical valves in recent studies. ${ }^{10-12}$ Our 10-year survival in the present study was at the lower end of survival compared with the previously reported mortality. The operative mortality was greater in the MVRb group before propensity matching, but this difference disappeared after cohort matching. Our operative mortality of $5.6 \%$ in the MVRm group and $8.0 \%$ in the MVRb group was lower than the reported result of $10 \%$ to $12.5 \%{ }^{12,13}$ The lower long-term survival in conjunction with the low operative mortality can be explained by our high-risk patient population. Our first choice for mitral valve regurgitation has been mitral valve repair. A high threshold for repair leaves high-risk candidates undergoing replacement. Also, $18.3 \%$ of the MVRm group and $22.0 \%$ of the MVRb group underwent concomitant CABG and $18.8 \%$ and $18.3 \%$ had undergone previous valve surgery, respectively. The survival benefit of the mechanical valve persisted after propensity matching. As mentioned, previous studies had reported similar mid- and long-term survival between mechanical and bioprostheses. We have discussed some of the variables that might have affected the outcomes in our study.

\section{Reoperation}

The reoperation rate was significantly greater in the MVRb group. SVD is a known Achilles heel of bioprosthetic valve that leads to reoperation. This is especially true in young patients. Ruel and associates ${ }^{10}$ published their data from 214 patients who had undergone MVR before the age of 60 years. The median interval to 


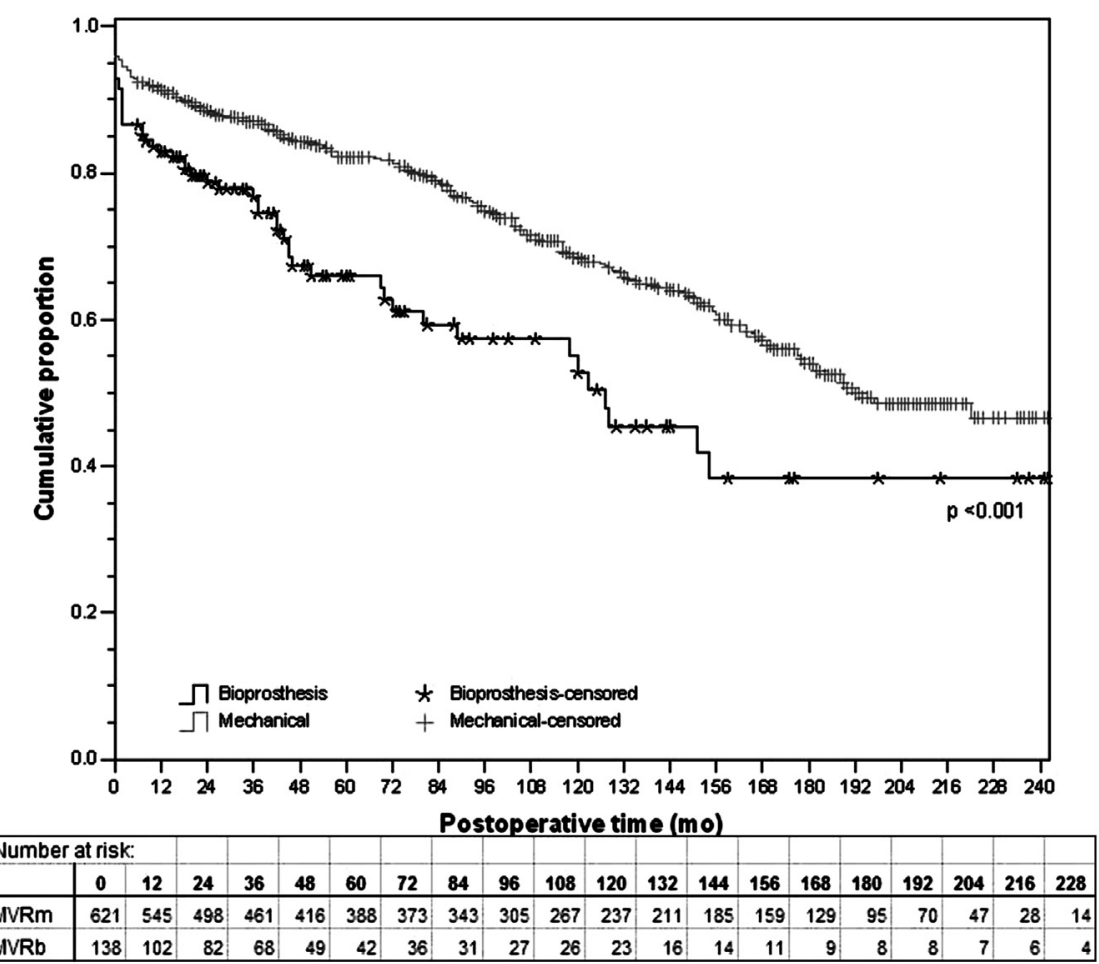

A

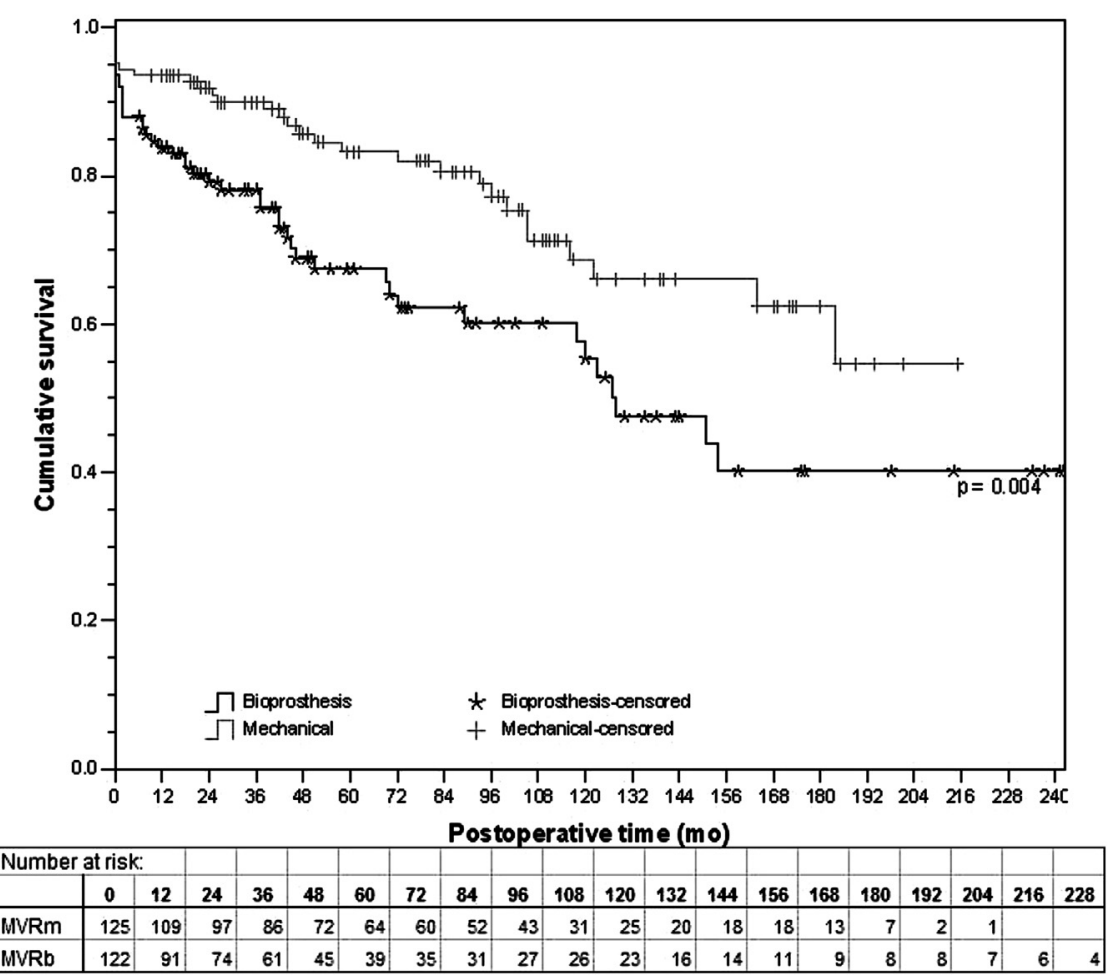

B

FIGURE 4. A, Kaplan-Meier curve of survival for all 761 patients. B, Kaplan-Meier curve of survival for 250-patient matched cohort. MVRm, Mitral valve replacement with mechanical valve; MVRb, MVR with bioprosthetic valve. 
TABLE 4. Cox regression analysis* for significant predictors of long-term mortality

\begin{tabular}{lccc}
\hline \multicolumn{1}{c}{ Variable } & $\boldsymbol{P}$ value & HR & $\mathbf{9 5 \%}$ CI \\
\hline Age & $\leq .001$ & 1.055 & $1.038-1.072$ \\
Perfusion time & $\leq .001$ & 1.005 & $1.004-1.006$ \\
Preoperative creatinine & $\leq .001$ & 1.351 & $1.275-1.431$ \\
Congestive heart failure & $\leq .002$ & 0.643 & $0.487-0.847$ \\
Urgent or emergent surgery & $\leq .006$ & 1.448 & $1.112-1.885$ \\
Previous CABG & $\leq .017$ & 1.587 & $1.088-2.316$ \\
Biologic valve & $\leq .017$ & 1.476 & $1.073-2.031$ \\
Previous valve surgery & $\leq .026$ & 1.439 & $1.045-1.982$ \\
Hypercholesterolemia & $\leq .050$ & 1.299 & $1.000-1.687$ \\
Nonsignificant variables tested & & & \\
Preoperative ejection fraction & $\leq .075$ & & \\
Endocarditis & $\leq .227$ & & \\
Female gender & $\leq .334$ & & \\
NYHA class & $\leq .391$ & & \\
History of cerebrovascular accident & $\leq .523$ & & \\
Body mass index & $\leq .617$ & & \\
Ischemic etiology & $\leq .784$ & & \\
Body surface area & $\leq .910$ & & \\
Hypertension & $\leq .949$ & & \\
\hline
\end{tabular}

Model performance: $-2 \log$ likelihood, 2945.533; chi-square, 285.699; degree of freedom, $7 ; P \leq .001$. $H R$, Hazard ratio; $C I$, confidence interval; $C A B G$, coronary artery bypass grafting; NYHA, New York Heart Association.

reoperation was 11.8 years with the tissue prostheses and 24.4 years with the mechanical prostheses. Jamieson and associates $^{8}$ reported an actual freedom from valve-related reoperation at 15 years for bioprosthetic valves of $<50 \%$ in patients $<60$ years and improved valve-related mortality in the mechanical group, except in those $>70$ years old. Our findings have confirmed these data, with bioprosthetic valves in the younger population associated with a greater risk of reoperation.

The valve type is considered an important risk factor for reoperation. The actual freedom from SVD has been reported to be $92 \%$ in those aged $>70$ years, $70 \%$ in those aged 61 to 70 years, and $56 \%$ in those aged $<60$ years at 15 years with the Carpentier-Edwards supra-annular (Edwards Lifesciences) porcine bioprosthesis. ${ }^{13}$ This is much lower than that for the new-generation valves such as the St Jude Medical Biocor valve (St Jude Medical), ${ }^{14}$ with actual freedom from SVD of $89 \%$ in those aged 61 to 70 years and $79 \%$ in those aged 51 to 60 years at 17 years. However, we examined our bioprosthetic valve type and found that the valve type was not related to an increased reoperation rate, even in the older generation valves.

Repeat replacement of the mitral valve is not without risk. Recent data have still reported mortality for repeat replacement of the mitral valve of $5 \%$ to $7 \%$, although this has decreased compared with previous reports. ${ }^{15-17}$ Hence, reoperation will likely affect the long-term survival of patients who require intervention.

\section{Bleeding}

Bleeding is considered a major problem for mechanical valves, because the mechanical valve requires lifelong anticoagulation. Previous randomized control studies ${ }^{5,6}$ and a recent retrospective study ${ }^{11}$ showed a greater bleeding rate with the mechanical valve. However, Badhwar and associates ${ }^{18}$ reported no difference in the bleeding rate between mechanical and bioprosthetic valves in their propensity matched analysis for patients $<65$ years who had undergone valve replacement (both aortic valve replacement and MVR included). They used a lower international normalized ratio goal (2.5 in the MVR group) and frequent international normalized ratio point-of-care checks at home, which might have decreased the incidence of bleeding. Excellent international normalized ratio management within their healthcare system and by caregivers could be 1 of possible explanations for the similar bleeding rate in the mechanical valve patients. Stassano and associates ${ }^{19}$ performed a randomized control study of patients aged 55 to 70 years who were undergoing aortic valve replacement. In that study, the mechanical valve resulted in a bleeding risk similar to that of the bioprosthetic valve. ${ }^{19}$ An increased number of patients in the bioprosthetic group required anticoagulation, especially with the longer followup period, for causes such as atrial fibrillation, transient ischemic attack, and chamber enlargement, although the exact number was not reported. ${ }^{19}$ Our follow-up showed that $25.8 \%$ of patients who received bioprosthetic valves required warfarin in the long term. This was likely because $18.4 \%$ of the patients who had received bioprosthetic valves had developed new-onset atrial fibrillation and might explain why the bioprosthetic group had a bleeding risk similar to that of the mechanical group.

The similar bleeding risks might explain why the survival was longer in mechanical group. The risk of valve implantation at a younger age is mainly bleeding with the mechanical valve and reoperation with the bioprosthetic valve. ${ }^{20}$ If the bleeding risk was similar between the 2 valve types, the bioprosthetic valve would have a greater risk because of reoperation.

\section{Thromboembolic Events}

Thromboembolic events occurred less frequently and resulted in fewer deaths in patients $<65$ years old. ${ }^{21}$ Also, other risk factors, such as coronary artery disease, atrial fibrillation, and poor left ventricular function, are less common in younger patients. Recent retrospective studies have shown a similar incidence of thromboembolic events between mechanical and bioprosthetic valves. ${ }^{10,11}$ The present study has confirmed that no increase occurred in thromboembolic events with the mechanical valves.

\section{Predictors of Long-Term Mortality}

In our Cox regression analysis, we identified multiple significant predictors of long-term mortality. Increased 
age, a long perfusion time, preoperative high creatinine, a history of congestive heart failure, urgent or emergent surgery, previous CABG and/or valve surgery, hypercholesterolemia, and biologic valve implantation were all statistically significant risk factors for patients undergoing MVR. The published data support our findings. Age at operation, coronary artery disease, class IV heart failure, reoperation, and renal disease were all reported risk factors for MVR for those aged $<60$ years. ${ }^{10,12}$ However, valve type was not reported to be a risk factor, differing from our results.

\section{Future Considerations for Transcatheter Procedures}

A previously placed bioprosthetic valve will allow VIV TMVR using the strut at reoperation. This technique has been welcomed with high expectations and has become 1 of the greatest reasons for receiving a bioprosthetic valve at a younger age. The consensus has been that patients will be able to receive a minimally invasive procedure without sternotomy and cardiopulmonary bypass when reoperation is required. However, only small case series have been reported regarding VIV TMVR to support this consensus. The largest series to date was presented by Dvir and associates at PCR London Valves 2012. From the global VIV registry, 91 patients who had undergone VIV TMVR were included in that study. They reported a 30 -day mortality of $11.9 \%$ and 1-year survival of $74.5 \%$. Although the future of this technique seems promising, the long-term outcomes and valve durability are still unknown, and larger series with longer follow-up are needed. The use of the VIV technique as a reason for implanting bioprosthetic valves in younger patients needs to be used with caution, especially with the shorter survival with bioprosthetic valves shown in the present study.

\section{Study Limitations}

The present study had limitations. This was a retrospective, single-center, observational cohort study. Underestimations of late events (reoperations, major bleeding and strokes) were possible. Paradoxically, our method has the potential to create a sampling bias that overestimates the long-term outcomes, because patients without events could be less well represented in our follow-up data. Also, some of the prostheses used in the present study are no longer commercially available, which could have altered the outcome. Despite our efforts to produce an evenly matched subset of comparable patients using a comprehensive step-wise logistic regression propensity score analysis, the potential always exists for confounders that are unmeasured and unknown. Also, we could not exclude the possibility that patients who were lost to follow-up had experienced subsequent events that were not captured in our study.

However, although our study was limited by its retrospective nature, we attempted to minimize the bias by using propensity matched analysis. This provided the balance of the 2 comparative groups with weighted effects of the variables and minimized the bias on assigning the valve types. The present study is 1 of the largest studies using propensity matched analysis in this specific age group.

\section{CONCLUSIONS}

Despite the recent trend of placing bioprosthetic valves in younger patients $<65$ years old, the present study has shown that patients who received a bioprosthetic valve have shorter survival than patients who received a mechanical valve for MVR. More reoperations occurred in the MVRb group, and the bleeding risk, which is considered a risk of mechanical valve implantation, was similar between the 2 groups. This might explain the poor outcome in this population. It is uncertain how the new bioprosthetic valve with longer durability and VIV TMVR will play role in the future. However, the results of the present study, 1 of the largest retrospective series on this subject with propensity matched analysis, have reaffirmed the recommendation to use the mechanical valve for MVR in those $<65$ years old.

\section{References}

1. Gammie JS, Sheng S, Griffith BP, Peterson ED, Rankin JS, O'Brien SM, et al. Trends in mitral valve surgery in the United States: results from the Society of Thoracic Surgeons Adult Cardiac Database. Ann Thorac Surg. 2009;87:1431-9

2. Kaneko T, Cohn LC, Aranki SF. Tissue valve is the preferred option for patients aged 60 and older. Circulation. 2013;128:1365-71.

3. Bonow RO, Carabello BA, Chatterjee K, de Leon AC, Faxon DP, Freed MD, et al. ACC/AHA 2006 guidelines for the management of patients with valvular heart disease. Circulation. 2006;114:e84-231.

4. Vahanian A, Alfieri O, Andreotti F, Antunes MJ, Baron-Esquivias G, Baumgartner H, et al. Guidelines on the management of valvular heart disease. Eur Heart J. 2012;33:2451-96.

5. Oxenham H, Bloomfield P, Wheatley DJ, Lee RJ, Cunningham J, Prescott RJ, et al. Twenty years comparison of a Bjork-Shiley mechanical heart valve with porcine bioprostheses. Heart. 2003;89:715-21.

6. Hammermeister K, Sethi GK, Henderson WG, Grover FL, Oprian C, Rahimtoola SH. Outcomes 15 years after valve replacement with a mechanical versus a bioprosthetic valve: final report of the Veterans Affairs randomized trial. J Am Coll Cardiol. 2000;36:1152-8

7. Masters RG, Haddad M, Pipe AL, Veinot JP, Mesana T. Clinical outcomes with the Hancock II bioprosthetic valve. Ann Thorac Surg. 2004;78:832-6.

8. Jamieson WR, von Lipinski O, Miyagishima RT, Burr LH, Janusz MT, Ling H, et al. Performance of bioprostheses and mechanical prostheses assessed by composites of valve-related complications to 15 years after mitral valve replacement. J Thorac Cardiovasc Surg. 2005;129:1301-8.

9. Berrebi AJ, Carpentier SM, Phan KP, Nguyen VP, Chauvaud SM, Carpentier A. Results of up to 9 years of high-temperature-fixed valvular bioprostheses in a young population. Ann Thorac Surg. 2001;71(5 Suppl):S353-5.

10. Ruel M, Chan V, Bedard P, Kulik A, Ressler L, Lam K, et al. Very long-term survival implications of heart valve replacement with tissue versus mechanical prostheses in adults <60 years of age. Circulation. 2007;116:S294-300.

11. Kulik A, Bedard P, Lam BK, Rubens FD, Hendry PJ, Masters RG, et al Mechanical versus bioprosthetic valve replacements in middle-aged patients Eur J Cardiothorac Surg. 2006;30:485-91

12. Cen YY, Glower DD, Landolfo K, Lowe JE, Davis RD, Wolfe WG, et al Comparison of survival after mitral valve replacement with biologic and mechanical valves in 1139 patients. J Thorac Cardiovasc Surg. 2001;122:569-77.

13. Jamieson WR, Gudas VM, Burr LH, Janusz MT, Fradet GJ, Ling H, et al. Mitral valve disease: if the mitral valve is not reparable/failed repair, is bioprosthesis suitable for replacement? Eur J Cardiothorac Surg. 2009;35:104-10.

14. Myken PS. Seventeen-year experience with the St. Jude Medical Biocor porcine bioprosthesis. J Heart Valve Dis. 2005;14:486-92. 
15. Potter DD, Sundt TM III, Zehr KJ, Dearani JA, Daly RC, Mullany CJ, et al. Risk of repeat mitral valve replacement for failed mitral valve prostheses. Ann Thorac Surg. 2004;78:67-72

16. Jamieson WR, Burr LH, Miyagishima RT, Janusz MT, Fradet GJ, Lichtenstein SV, et al. Reoperation for bioprosthetic mitral structural failure: risk assessment. Circulation. 2003;108:S98-102.

17. Cohn LH, Aranki SF, Rizzo RJ, Adams DH, Cogswell KA, Kinchla NM, et al. Decrease in operative risk of reoperative valve surgery. Ann Thorac Surg. $1993 ; 56: 15-20$

18. Badhwar V, Ofenloch JC, Rovin JD, van Gelder HM, Jacobs JP. Noninferiority of closely monitored mechanical valves to bioprostheses overshadowed by early mortality benefit in younger patients. Ann Thorac Surg. 2012;93: 748-53

19. Stassano P, DiTommaso L, Monaco M, Iorio F, Pepino P, Spampinato N, et al. Aortic valve replacement: a prospective randomized evaluation of mechanical versus biological valves in patients ages 55 to 70 years. J Am Coll Cardiol. 2009;54:1862-8.

20. Rahimtoola SH. Choice of prosthetic heart valve in adults: an update. J Am Coll Cardiol. 2010;55:2413-26.

21. Jamieson WR, Miyagishima RT, Grunkemeier GL, Germann E, Henderson C, Lichtenstein SV, et al. Bileaflet mechanical prostheses for aortic valve replacement in patients younger than 65 years and 65 years of age or older: major thromboembolic and hemorrhagic complications. Can J Surg. 1999;42:27-36.

Access to The Journal of Thoracic and Cardiovascular Surgery Online is reserved for print subscribers!

Full-text access to The Journal of Thoracic and Cardiovascular Surgery Online is available for all print subscribers. To activate your individual online subscription, please visit The Journal of Thoracic and Cardiovascular Surgery Online, point your browser to http://www.mosby.com/itcvs, follow the prompts to activate your online access, and follow the instructions. To activate your account, you will need your subscriber account number, which you can find on your mailing label (note: the number of digits in your subscriber account number varies from 6 to 10). See the example below in which the subscriber account number has been circled:

\section{Sample mailing label}

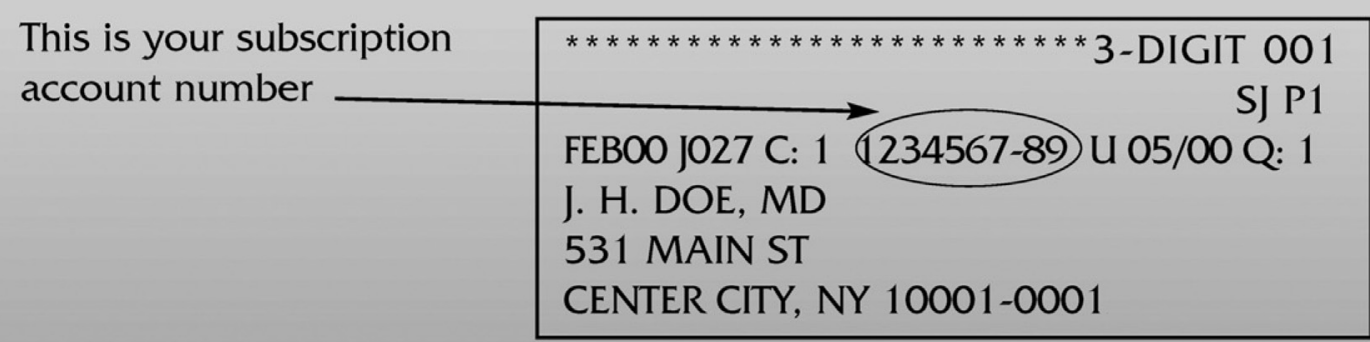

Personal subscriptions to The Journal of Thoracic and Cardiovascular Surgery Online are for individual use only and may not be transferred. Use of The Journal of Thoracic and Cardiovascular Surgery Online is subject to agreement to the terms and conditions as indicated online. 